\title{
Calcified thoracic disc with herniation of the nucleus pulposus in a child
}

\author{
D. Schapira, ${ }^{1}$ D. Goldsher, ${ }^{2}$ M. Nahir ${ }^{1}$ and Y. Scharf ${ }^{1}$ \\ Departments of ${ }^{1}$ Rheumatology and ${ }^{2}$ Diagnostic Radiology, Rambam Medical Center, Faculty of Medicine, \\ Technion-Israel Institute of Technology, Haifa, Israel.
}

\begin{abstract}
Summary: A twelve year old boy presented with acute cervical and interscapular pain, torticollis and low grade fever. The neurological examination was normal. Roentgenograms showed calcification of the $T_{3}-T_{4}$ disc and posterior herniation of the nucleus pulposus was demonstrated by computed tomography. A rapid and dramatic amelioration of the symptoms was produced by treatment with a non-steroidal anti-inflammatory drug.

Intervertebral disc calcification in childhood is an uncommon and usually benign condition. Its clinical and radiological features are discussed in the light of the relevant literature.
\end{abstract}

\section{Introduction}

In adults, calcification of the intervertebral discs is common and has been associated with advanced age, with affections like ochronosis, haemochromatosis, chondrocalcinosis, hyperparathyroidism, ankylosing spondylitis, poliomyelitis and amyloidosis. In most of the cases, both the annulus fibrosus and the nucleus pulposus are calcified and chronic back pain is the common clinical finding. ${ }^{1}$ In children, calcification of the nucleus pulposus is linked to a well defined benign and self-limited clinical condition, usually responsive to conservative treatment. The following case is representative. The diagnostic value of the computed tomography and the need for a nonaggressive therapeutic management are emphasized.

\section{Case report}

A 12 year old boy presented with a four day history of neck and upper back pain radiating to the shoulders. There was no history of previous infectious disease or of direct trauma. He was slightly febrile. Physical examination demonstrated a torticollis with contraction of the sternocleidomastoid muscle, severe limitation of the neck motion and muscle spasm in the cervical and upper

Correspondence: D. Schapira M.D., The B. Shine Department of Rheumatology, Rambam Medical Center, Haifa 3524, Israel

Accepted: 25 August 1987 thoracic region. This area was very sensitive on palpation. There was no neurological deficit. Laboratory investigation showed a sedimentation rate of $30 \mathrm{~mm} / \mathrm{h}$ and a white cell count os $12.9 \times 10^{9} / 1$. Serum biochemistry was normal and serological tests for rheumatic diseases were negative.

Roentgenograms revealed calcification in the space between the third and the fourth thoracic vertebrae (Figure 1). Computerized tomography of the upper thoracic spine revealed calcified nucleus pulposus with centrolateral herniation into the spinal canal (Figures 2 and 3). The diagnosis of herniated calcified thoracic disc was established. Treatment with a non-steroidal anti-inflammatory drug (ibuprofen $400 \mathrm{mg}$ twice a day) brought a rapid and dramatic amelioration with disappearance of the pain and limitation after three days. The drug was discontinued and the child remained asymptomatic during the next two months. Later he was lost to follow-up.

\section{Discussion}

Intervertebral disc calcification in children is an uncommon condition. Since Baron's first description $^{2}$ fewer than 130 cases have been reported. ${ }^{3-8}$ The calcification occurs in the nucleus pulposus. The discs affected are cervical, thoracic and lumbar, in decreasing order and in one third of the cases several discs are involved. ${ }^{9}$ This condition

(C) The Fellowship of Postgraduate Medicine, 1988 


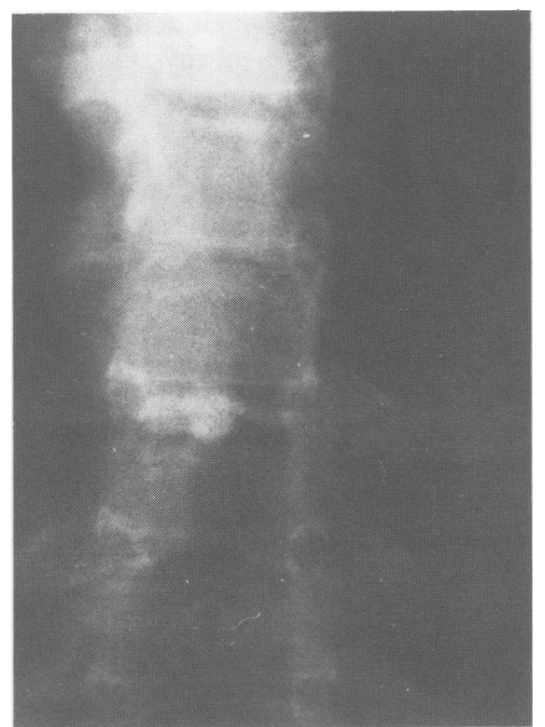

Figure 1 Plain radiograph of the thoracic spine showing calcification of the $T_{3}-T_{4}$ interspace. The structure of the spine is otherwise normal.

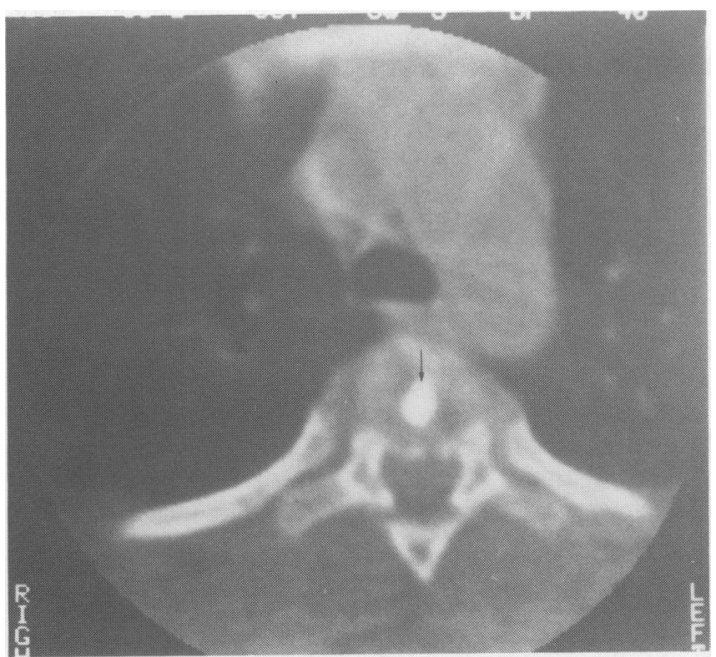

Figure 2 Computed tomography of the $T_{3}-T_{4}$ intervertebral space showing dense irregular calcification of the nucleus pulposus.

is associated with clinical signs and symptoms which usually occur when the calcification is cervical and persist for a short period of time, rarely beyond a few weeks. ${ }^{10-11}$ The calcifications may occur at any age during childhood but are more frequent between the ages of 5 and 10 years. A male predominance was noted. ${ }^{12}$

The aetiology is unknown and no metabolic basis

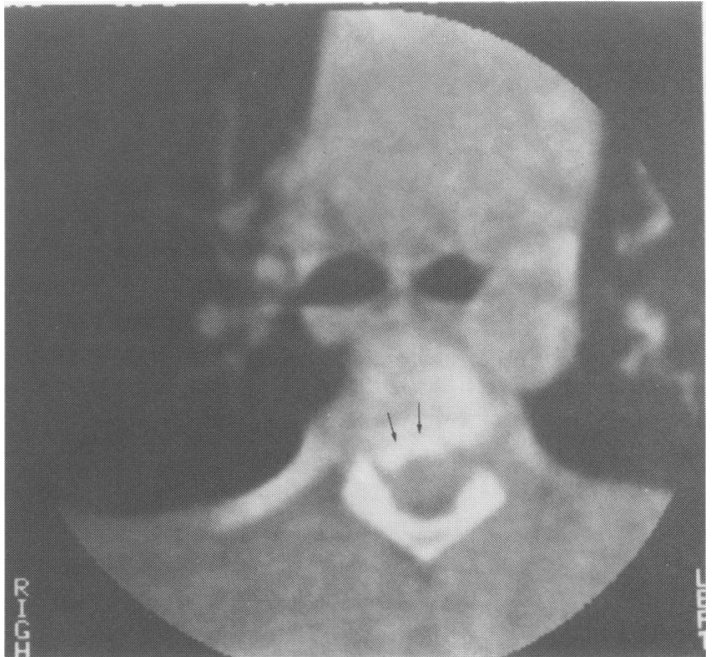

Figure 3 Centrolateral posterior herniation of the calcified disc material into the spinal canal.

has been found. Trauma has been implicated in some cases but usually the chronological relation is uncertain and no precipitating event can be found. In a few cases disc calcifications, mainly thoracic, have been associated with congenital cardiac lesions, infection, bilateral cataracts and congenital bony malformation. ${ }^{13,14}$ In childhood the differential diagnosis includes infectious discitis, non-specific inflammatory conditions of the disc space (also referred to as discitis or spondylarthritis) ${ }^{15}$ and vitamin $D$ intoxication in which the annulus fibrosus is calcified. ${ }^{16}$ If clinically suspected, meningitis must be confirmed by a lumbar puncture.

Clinical and radiological criteria have been proposed for the definition of the intervertebral disc calcification syndrome: ${ }^{17}$

1. Severe focal or referred pain of acute onset.

2. Painful limitation of the spine motion, muscle spasm and tenderness and sometimes persistent torticollis.

3. Local signs of inflammation and in some patients systemic fever, leucocytosis, and an elevated sedimentation rate. They are probably concomitant with the absorption of the calcification.

4. The radiological presence of an intervertebral disc calcification, central or with anterior, lateral or posterior protrusion, as shown by plain X-rays, tomography and myelography. Bone scintigraphy may confirm the location of the lesion and help to exclude infections and malignant conditions. Computed tomography has proved to be a promising non-invasive measure in the diagnosis of 
the extruded calcified disc. ${ }^{18}$ This is clearly demonstrated by our case in which to our knowledge computerized tomography was used for the first time to diagnose calcified disc herniation in a child.

5. Limitation of the disease to the paediatric age group only.

6. Self-limited clinical course.

Prognosis is good. In the majority of the patients the symptoms disappear spontaneously within a few weeks. The calcification is also self-limited and disappears after a few months. Sometimes it may persist for several years but the children are asymptomatic. However, long term (2-9 years) clinical and radiological findings have been reported. ${ }^{10,17,19}$ They include recurrent acute inflammatory episodes and persistent back pain, residual calcifications, flattening of the vertebral bodies, osteophytes and disc space narrowing. There is a certain similarity with cases of calcified

\section{References}

1. Weinberger, A. \& Myers, A.R. Intervertebral disc calcifications in adults: a review. Semin Arth Rheum 1978, 8: 69-75.

2. Baron, A. Uber eine neue Erkrankung die Wirbelsaüle. Jahrb Kinderheilk 1924, 104: 357-360.

3. Walker, C.S. Calcification of the intervertebral discs in children. J Bone Joint Surg 1954, 36-B: 601-605.

4. Rechtman, A.M., Hermel, M.B., Albert, S.M. \& Boreadi, A.G. Calcification of the intervertebral disc: disappearing, dormant and silent. Clin Orthop 1956, 7: 218-231.

5. Bjelkhagen, I. \& Gladnikoff, H. Calcified disc protrusion in children. Acta Radiol 1957, 48: 151-155.

6. Henry, M.J., Grimes, H.A. \& Lane, J.W. Intervertebral disc calcification in childhood. Radiology 1967, 89: 81.

7. Hayem, F., Fortier-Beaulieu, M., Masselot, J. \& Taillemite, J.L. La discopathie calcifiante de l'enfant. A propos de quatre observations. Ann Radiol 1972, 15: $96-104$.

8. Blomquist, H.C., Lingvist, M. \& Mattson, S. Calcification of intervertebral discs in childhood. Pediatr Radiol 1979, 8: 23.

9. Froment, C. \& Husson, J. La nucléopathie calcifiante de l'enfant. Sem Hôp Paris 1986, 62: 117-121.

10. Newton, T.H. Cervical intervertebral disc calcification in childhood. J Bone Joint Surg 1958, 40-A: 107-118.

11. Melnick, J.C. \& Silverman, F.M. Intervertebral disc calcification in childhood. Radiology 1963, 80: 399408.

12. Morris, J. \& Niebauer, J. Calcification of the cervical intervertebral disc. Am J Dis Child 1963, 106: 295300.

13. Lasserre, C. \& Phellipot, G. Discite calcifiante intervertebrale. Rev Orthop 1947, 33: 494-500. bursitis and tendonitis in which acute inflammation is usually - though not always - followed by clinical remission, with diminution or disappearance of the calcifications. ${ }^{20-22}$

In spite of the fact that in the acute phase herniation of the calcified nucleus pulposus through the annulus fibrosus has been demonstrated radiologically or during surgery, only a few cases present objective neurological findings. Spinal cord compression $^{23}$ and transverse myelitis ${ }^{10}$ have been reported.

Conservative treatment is the rule and prognosis is excellent even when herniation of the nucleus pulposus occurs. ${ }^{24}$ Temporary restriction of vigorous physical activities, analgesics and nonsteroidal anti-inflammatory drugs are very efficient. There is no justification for antibiotics, physical therapy and long term immobilization. Surgery must be reserved only for those patients in whom there is evidence of progressive cord compression.

14. Silverman, F.M. Calcification of the intervertebral disks in childhood. Radiology 1954, 62: 801-815.

15. Spiegel, P.G., Kengla, K.W., Isaacson, A.S. \& Wilson, J.C. Intervertebral disc-space inflammation in children. J Bone Joint Surg 1972, 54-A: 284-295.

16. Swick H.M. Calcification of intervertebral discs in childhood. J Pediatr 1975, 86: 364-369.

17. Eyring, E.J., Peterson, C.A. \& Bjorson, D.R. Intervertebral disc calcification in childhood: a distinct clinical syndrome. J Bone Joint Surg 1964, 46-A: 1432-1441.

18. Hochman, M.S., Pena, C. \& Ramirez, R. Calcified herniated thoracic disc diagnosed by computerized tomography. J Neurosurg 1986, 52: 722-723.

19. Morris, I.M. \& Sheppard, L. The persistence of clinical and radiological features after intervertebral disc calcification of childhood. Br $J$ Rheumatol 1986, 25: 219-221.

20. Goldenberg, R.R. \& Leventhal, G.S. Supratrochanteric calcifications. J Bone Joint Surg 1936, 18: 205-211.

21. Mathews, P.P. \& Leyshon, R. Acute calcification in tennis and golfer's elbow. Rheumatol Rehabil 1980, 19: 151-153.

22. McKendry, R., Unthoff, H.K., Sarkar, K. \& Hyslop, P. Calcifying tendinitis of the shoulder: prognostic value of clinical, histologic and radiologic features in 57 surgically treated cases. $J$ Rheumatol 1982, 9: 7580.

23. Peck, F.C. Jr. A calcified thoracic intervertebral disk with herniation and spinal compression in a child. Case report. J Neurosurg 1957, 14: 105-109.

24. Mainzer, F. Herniation of the nucleus pulposus. A rare complication of intervertebral disk calcification in children. Radiology 1973, 107: 167-170. 